\title{
A Method for Deriving Quantum Dynamic Equations from Classical Mechanics
}

\author{
Hua Ma \\ The College of Science, Air Force University of Engineering, Xi'an, People's Republic of China
}

Email address:

mahuar@xjtu.edu.cn

\section{To cite this article:}

Hua Ma. A Method for Deriving Quantum Dynamic Equations from Classical Mechanics. American Journal of Physics and Applications. Vol. 5, No. 6, 2017, pp. 80-83. doi: 10.11648/j.ajpa.20170506.11

Received: August 14, 2017; Accepted: September 6, 2017; Published: October 11, 2017

\begin{abstract}
Based on the operator theories and Hamiltonian canonical equation, an operator based quantum dynamics equation is established, which has the same effect as the Hamiltonian equation in describing the state evolution of quantized dynamical systems. As the reasonable verification of this equation, Schrodinger equation can be derived theoretically, and the variational principle properties of quantum mechanics are revealed. This work will help to promote the development of quantum theory and to perfect the axiomatic system of quantum mechanics.
\end{abstract}

Keywords: Classical Mechanics, Quantum Mechanics, Variational Principle, Hamiltonian Canonical Equation, Schrodinger Equation, Operator Theory

\section{Introduction}

The variational principle is the general principle of scientific principles in calculus variation, and thus for the discovery of functional extremes, depending on the development of these functions [1-3]. All laws of physics can be mathematically described by self adjoint operator as a variational principle. In classical mechanics, these expressions are called Hamiltonian principles, which were first proposed by William Ron Hamilton in 1833. Hamiltonian mechanics is a theory developed as a new development of classical mechanics. It predicts the same result of non-classical mechanics. It employs a variety of mathematical formalisms that provide a more abstract understanding of the theory. It is pointed out that the dynamics of a physical system is a single function basis by a functional variational problem on Lagrange, which contains information about all of the physical system and the force acting on it $[1,4,5]$.

Quantum mechanics is a branch of physics as the fundamental theory of nature at small scales and low energy levels of atoms and subatomic particles, which gradually arose from Max Planck's solution to the black-body radiation problem and Albert Einstein's interpretation of the photoelectric effect. Early quantum theory was profoundly reconceived in the mid-1920s. The reconceived theory is formulated in various specially developed mathematical formalisms. The sign of perfection and trend of quantum mechanics is the introduction of wave function, Max Born's statistical interpretation, and the establishment of Schrodinger equation $[6,7]$.

Schrodinger equation is the mathematical equation that describes the way that the quantum wave function, which plays the same role for a quantum system as that Newton's laws of motion play in the mechanics of everyday objects. Schrodinger equation comes from Erwin Schrodinger, when he first derived these formulas and applied them to the behavior of an electron. As a mathematical formulation for studying quantum mechanical systems, it is considered a central result in the study of quantum system and its derivation was a significant landmark in developing the theory of quantum mechanics [8-10].

Today, Quantum mechanics has entered the axiomatization system, especially having perfect combinations with classical mechanics. In recent years, many scholars have studied and put forward a rich new ideas about the derivation, explanation, solution, and the expansion of applications focusing on the quantum dynamic equations [11-14]. However, how the Schrodinger equation is established is still a mystery, and still a suspense about constructing the axiomatic system of quantum mechanics. Historically, many physicists tried to give different derivations: Erwin Schrodinger established it by operating harmonic equation, Heisenberg explained its 
variational nature by matrix mechanics, Richard Feynman proved the equivalence between it and path integral mechanics, and later it was attempted to derive by group theory $[6-8,10]$. All these works contain the components of conjecture and analogy, and there are still some deficiencies in the construction of axiomatic system of Quantum mechanics. It's really hard to theoretically deduce the formula, just as Richard Feynman says: It is not possible to derive it from anything you know. It came out of the mind of Schrödinger [15].

After years of thinking, I have finally found a scheme for deriving the Schrodinger equation, which is based on the quantization hypothesis and the Hamilton principle. In the derivations, it was found that the Hamiltonian canonical equation can be handled by operator, so as to obtain a generalized dynamics equation based on operator description, which has a broader physical connotation and can be used as the basis of the Schrodinger equation. In the follows: firstly, from Hamiltonian mechanics, establish the general quantum dynamics equation using operator methods and quantization hypothesis; then, derive the expression of momentum operator based on the quantum hypothesis; and finally, apply the momentum operator expression to the general quantum dynamic equation to derive Schrodinger equation.

\section{Deduction of the General Quantum Dynamic Equation}

\subsection{Quantization Hypothesis}

Quantization hypothesis, established by Max Planck, Albert Einstein, and De Broglie et al., which can be summarized as: for a quantum system, energy, momentum and other quantities are restricted to discrete values, and objects have characteristics of wave-particle duality [6, 7]. In following derivations, some basic ideas were adopted as the foundations. First, the assumption of quantum states: Quantum systems behave some states, which are represented by wave functions. Second, the statistical explanation on wave functions: Wave functions have complex-valued probability amplitudes, from which the probabilities for the possible results of measurements made on the system can be calculated. Third, the mathematical approach based on the operator theory: Any observable physical quantities, i.e. position, momentum, energy, angular momentum etc., should be associated with a self adjoint linear operator [16]. The operators must yield real eigenvalues, since they are values that may come up as the result of the experiment. As the results, the physical operators must be Hermitian in Hilbert space.

In accordance with the above assumptions and requirements, the wave function must be square integrable meaning in region $\mathrm{R}$ with $\mathrm{N}$-dimensional coordinate vector $q$ :

$$
\int_{\mathrm{R}}|\psi(q, t)|^{2} d^{\mathrm{N}} q=\int_{\mathrm{R}} \psi^{*}(q, t) \psi(q, t) d^{\mathrm{N}} q<\infty
$$

And the normalization condition results in:

$$
\int_{\mathrm{R}} \psi^{*}(q, t) \psi(q, t) d^{\mathrm{N}} q=1
$$

The ideas expressed by Eqs. (1) and (2) will be used as the basis for the following derivations.

\subsection{The Time Dependent Differential Form of Hamiltonian Canonical Equation}

In Hamiltonian mechanics, a classical physical system is described by a set of canonical coordinates $(q, p)$ and Hamiltonian $H$, where $q$ and $p$ are default as vectors in full text The time evolution of a dynamical system is uniquely defined by Hamiltonian canonical equation $[1,4,5]$ :

$$
\frac{d}{d t} p=-\frac{\partial}{\partial q} H, \frac{d}{d t} q=\frac{\partial}{\partial p} H
$$

In actual physical processes, momentum and coordinates are functions of time:

$$
p=p(t), q=q(t)
$$

So, Hamiltonian $H$ can be expressed as:

$$
H=H(q(t), p(t))
$$

And thus its differential is:

$$
d H=\frac{\partial H}{\partial q} d q+\frac{\partial H}{\partial p} d p=\left(\frac{\partial H}{\partial q} \frac{d q}{d t}+\frac{\partial H}{\partial p} \frac{d p}{d t}\right) d t
$$

Taking Hamiltonian canonical Eq. (3) into Eq. (6), it reads:

$$
d H=\left(-\frac{d p}{d t} \frac{d q}{d t}+\frac{d q}{d t} \frac{d p}{d t}\right) d t
$$

\subsection{Derivation of the Quantum Dynamical Equation in the Form of Operator}

Introducing operators $q \rightarrow \hat{q}$ and $p \rightarrow \hat{p}$ into Eq. (7), and using $\cong$ denote the equivalence relation between two operators, which means that the two operators with different expressions have the same effect when they act on a wave function based on the quantum hypothesis. So, operator based Eq. (7) is:

$$
d \hat{H} \cong\left(-\frac{d \hat{p}}{d t} \frac{d \hat{q}}{d t}+\frac{d \hat{q}}{d t} \frac{d \hat{p}}{d t}\right) d t
$$

The right term of Eq. (8) can be simplified as:

$$
\left(-\frac{d \hat{p}}{d t} \frac{d \hat{q}}{d t}+\frac{d \hat{q}}{d t} \frac{d \hat{p}}{d t}\right) d t=\frac{d^{2}}{d t^{2}}(\hat{q} \hat{p}-\hat{p} \hat{q}) d t=\frac{d^{2}}{d t^{2}}[\hat{q}, \quad \hat{p}] d t
$$

where [,] is commutation operator. Bringing Eq. (9) into Eq. (8), and performing integrate operation on both sides of Eq. (8):

$$
\int d \hat{H} \cong \int \frac{d^{2}}{d t^{2}}[\hat{q}, \quad \hat{p}] d t=\int \frac{d}{d t}\left(\frac{d}{d t}[\hat{q}, \quad \hat{p}]\right) d t
$$


Then, comes to:

$$
\hat{H} \cong \frac{d}{d t}[\hat{q}, \quad \hat{p}]
$$

The formula (11) means that, Hamiltonian operator $\hat{H}$ is equivalent to the operator $\frac{d}{d t}\left[\begin{array}{l}\hat{q}, \hat{p}\end{array}\right]$. So, when they act on the wave function $\psi(q, t), \cong$ in formula $(11)$ can be replaced by $=$, that is

$$
\hat{H} \psi(q, t)=\frac{d}{d t}[\hat{q}, \quad \hat{p}] \psi(q, t)
$$

From Eqs. (3) to (12), no constraints are applied, which is in fact equivalent to the Hamiltonian canonical equation. Hamiltonian canonical equation is the general dynamic equation for motion systems. The equation (12) should be the general dynamic equation for quantum systems, because it is established by introducing the operator method and the quantization hypothesis on the basis of the Hamiltonian canonical equation.

\section{Deduction of Schrodinger Equation}

Based on the quantization hypothesis, momentum operator can be derived, and Schrodinger equation can be established from Eq. (12).

\subsection{Derivation of Momentum Operator}

According to quantization hypothesis, the energy and the momentum for a quantum system can be expressed as:

$$
E=\hbar \omega, p=\hbar k
$$

where $E$ and $p$ is energy and momentum under particle view, respectively; $\omega$ and $k$ is the angular frequency and wave vector under wave view, respectively; $\hbar$ is Planck's constant divided by $2 \pi$.

The simplest wave function is a plane wave with the form in position space:

$$
\psi(r, t)=\mathrm{A} e^{-\mathrm{i}(\omega t-k \cdot r)}
$$

where $\mathrm{A}$ is the amplitude, $k$ is the wave vector, and $\omega$ is the angular frequency, of the plane wave. According to Quantization hypothesis, the wave vector of a free particle with energy $E$ and momentum $p$ is:

$$
\psi(r, t)=\mathrm{A} e^{-\frac{\mathrm{i}}{\hbar}(E t-p \cdot r)}
$$

The expectation value is the average measurement of an observable physical quantity, so the momentum $p$ can be obtained by $\hat{P}$ acting on the wave function in region $\mathrm{R}$ :

$$
p=\int_{\mathrm{R}} \psi^{*}(r, t) \hat{P} \psi(r, t) d^{3} r
$$

Bringing Eq. (15) into Eq. (16), it reads:

$$
p=\int_{\mathrm{R}}\left[\mathrm{A}^{*} e^{\frac{\mathrm{i}}{\hbar}(E t-p \cdot r)} \hat{P}\left(\mathrm{~A} e^{-\frac{\mathrm{i}}{\hbar}(E t-p \cdot r)}\right)\right] d^{3} r
$$

Taking observation on Eq. (17), it can be concluded that: if and only if

$$
\hat{P}=-\mathrm{i} \hbar \nabla
$$

the Eq. (17) can hold on, where $\nabla$ is the Hamiltonian operator. Adopting the principle of generality, the expression of the momentum operator as expression (18) can be generalized to a general form, which is useful in the following deduction of Schrodinger equation.

\subsection{Derivation of Schrodinger Equation}

In real manifold space, the operator of $q$ is $\hat{r}$, and $\left[\begin{array}{ll}\hat{r}, \hat{p}\end{array}\right]$ can be derived based on Eq. (18):

$$
\left[\begin{array}{ll}
\hat{r}, & \hat{p}
\end{array}\right]=\mathrm{I}(\mathrm{i} \hbar)
$$

where I is unit matrix. In the nonrelativistic case, the commutation operator of coordinate and momentum is associated with scalar wave functions, so matrix I can be substituted by 1 , here:

$$
\frac{d}{d t}[\hat{r}, \quad \hat{p}]=\frac{d}{d t}(\mathrm{i} \hbar)=\mathrm{i} \hbar \frac{d}{d t}
$$

Taking Eq. (20) into Eq. (12) with considering $\frac{d}{d t} \rightarrow \frac{\partial}{\partial t}$ for $\psi(r, t)$, get:

$$
\hat{H} \psi(r, t)=\mathrm{i} \hbar \frac{\partial}{\partial t} \psi(r, t)
$$

which is just the Schrodinger equation.

To be noted that, in the above derivations, there are two places where the theory is not rigorous: one is the expression of the momentum operator, and the other one is how the commutation matrix of momentum and coordinate is reduced to scalar form. However, the conclusions of the two derivations are entirely correct and have been confirmed by history and experiments, so it is reasonable to establish Schrodinger equation through Eq. (12).

\section{Conclusion}

By introducing the operator method and the quantization hypothesis, the general dynamic equation for quantum systems was established based on Hamiltonian canonical equation, so it can be used as the basic laws of motion for quantum systems. Compared with the Schrodinger equation, this equation has a more general form, and the Schrodinger equation can be derived from which. It not only indicates the intrinsic unity of classical mechanical system and quantum mechanics, but also reveals that the current quantum 
mechanics has more deep rules needed to be further explored. For instance, in some other quantum systems, the motion law of the quantum system is no longer described by the Schrodinger equation if the commutation relation between position and momentum is in other forms.

By analyzing the momentum operator based on the quantization hypothesis, Schrodinger equation can be derived from the general dynamic equation, certifying the validity of the general quantum mechanical formula established here. This work reveals the nature of the variational principle of the dynamics of the quantum system, and thus promotes the perfection of the axiomatic system of quantum mechanics. The establishment of the general quantum dynamic equation from classical mechanics, as well as the application in deriving Schrodinger equation, are expected to open up a new dynamics system, using which to describe the evolution law of quantized and non-quantized dynamical systems in a unified way.

\section{References}

[1] https://en.wikipedia.org/wiki/Classical_mechanics.

[2] C Lanczos, The Variational Principles of Mechanics (University of Toronto Press, Toronto, 1964).

[3] Cassel, Kevin W., Variational Methods with Applications in Science and Engineering (Cambridge University Press, 2013).

[4] Landau LD and Lifshitz EM, Mechanics (Pergamon Press, 3rd ed., pp. 2-4, 1976).
[5] Hand L. N., Finch J. D., Analytical Mechanics (Cambridge University Press, 2008).

[6] Kragh, Helge, Quantum Generations: A History of Physics in the Twentieth Century (Princeton University Press, p. 58, 2002).

[7] Mehra J., Rechenberg H., The historical development of quantum theory (New York: Springer-Verlag, 1982).

[8] Schrödinger E., Physical Review. 28 (6): 1049-1070 (1926).

[9] Griffiths, David J., Introduction to Quantum Mechanics (Prentice Hall, 2nd ed., 2004).

[10] Sakurai J. J., Modern Quantum Mechanics (Massachusetts: Addison-Wesley, p. 68, 1995).

[11] J. Köppe, W. Grecksch, W. Paul, "Derivation and application of quantum Hamilton equations of motion", Annalen Der Physik, 2016, 529 (3): 1600251.

[12] VG Zelevinsky, "Microscopic derivation of a quantum hamiltonian for adiabatic collective motion", Nuclear Physics A, 2016, 337 (1): 40-76.

[13] J Zheng-Johansson, "PI Johansson, Inference of Schrödinger Equation from Classical-Mechanics Solution", Physics, 2006, 17 (3): 240-244.

[14] MA Ajaib, "A Fundamental Form of the Schrodinger Equation", Foundations of Physics, 2015, 45 (12): 1586-1598.

[15] T. Hey, P. Walters, The New Quantum Universe (Cambridge University Press, 2009).

[16] D. McMahon, Quantum Mechanics Demystified (Mc Graw Hill (USA), 2006). 